\title{
THE SET OF ALL COVERING SPACES
}

\section{J. GIL DE LAMADRID AND J. P. JANS ${ }^{1}$}

1. Introduction. Recently, Lee extended the definition of covering space to spaces which are not necessarily locally connected [2]. He showed that many of the classical results [1, Chapters II, VI-X] on covering spaces and simple connectedness can be extended to this wider class of spaces. In this paper, using a modification of Lee's definition, we consider the structure of the set $C(X)$ of "all" covering spaces of a space $X$. We put an order on $C(X)$ and consider conditions under which $C(X)$ has a last element, a universal covering space. We shall make a distinction between simply connected covering space and universal covering space. Although simply connected covering spaces are universal, the converse is an open question. We also show that a universal covering space, if it exists, is unique. The last section gives examples.

We call the pair $(X, f)$ a covering space of $X_{0}$ if

1. $X$ is connected and $f$ is a continuous map of $X$ onto $X_{0}$.

2. For every $x \in X_{0}$ there exists an open connected neighborhood $U$ of $x$ such that $f^{-1}(U)$ is the union of disjoint open sets $U^{*}$ with the property that $f \mid U^{*}$ is a homeomorphism onto $U$. Equivalence of two covering spaces is defined in the usual way $[1$, p. 43]. As in [2], we say that $U$ is evenly covered by $f$ and the sets $U^{*}$ are the even portions of $f^{-1}(U)$. Note that evenly covered sets are assumed connected. Our definition of covering space is more restrictive than that of [2] but more general than that of $[1, \mathrm{p} .40]$. The space $X$ is simply connected if every covering space $(Y, g)$ of $X$ is equivalent to the trivial covering space $(X, i), i$ the identity map on $X$.

Let $C\left(X_{0}\right)$ be the set of "all covering spaces" of $X_{0}$. Such a set exists in the sense of $[1, \mathrm{p} .44]$. If $(X, f)$ is a covering space of $X_{0}$ and $g: Y \rightarrow X_{0}$ is continuous, we shall say that $g$ can be lifted with respect to $f$ if there exists a continuous mapping $\bar{g}: Y \rightarrow X$ such that $f \circ \bar{g}=g$. The mapping $\bar{g}$ is called the lifting of $g$.

Cancellation Lemma 1.1. Let $(X, f)$ be a covering space of $X_{0}$. If $g$ and $h$ are continuous functions from a connected space $Y$ into $X$ such that $g(y)=h(y)$ for some point $y$ in $Y$ and $f \circ g=f \circ h$ then $g=h$.

The proof is as in $[1$, p. 51].

Received by the editors July 18, 1958.

1 The second author was supported, in part, by the National Science Foundation. 
2. Uniformly covered spaces. We call the connected open subset $U$ of $X_{0}$ uniformly evenly covered if for every element $(X, f)$ of $C\left(X_{0}\right)$, $U$ is evenly covered by $f$. The space $X_{0}$ is a uniformly covered space if every point of $X_{0}$ lies in a uniformly evenly covered open set. Note that spaces satisfying the first half of the conclusion of Lemma 4.1 of [2] are uniformly covered.

Lemma 2.1. If $X_{0}$ is a uniformly covered space and if the diagram

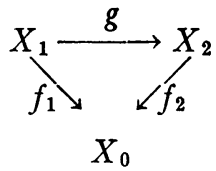

is commutative where $f_{1}$ and $f_{2}$ are covering maps and $g$ is continuous, then $g$ is also a covering map.

Proor. The assumption of uniform even covering is unnecessary if $X_{0}$ is locally connected; we only use the fact that $f_{1}$ and $f_{2}$ evenly cover the same connected neighborhoods.

If the open set $U$ is evenly covered by $f_{1}$ and $f_{2}$, let $U_{1}$ be a component of $f_{1}^{-1}(U)$, and let $U_{2}$ be the component of $f_{2}^{-1}(U)$ that contains $g\left(U_{1}\right)$. Then $g: U_{1} \rightarrow U_{2}$ equals $\left(f_{2} \mid U_{2}\right)^{-1} \circ f_{1}$, and is a homeomorphism. It follows easily that $g\left(X_{1}\right)$ is open, and that $g$ is a covering of $g\left(X_{1}\right)$. Moreover, $g\left(X_{1}\right)$ is closed; Let $x_{2} \in X_{2}$, let $U$ be as before with $f_{2}\left(x_{2}\right) \in U$, and let $U_{2}$ be the component of $f_{2}^{-1}(U)$ containing $x_{2}$. Suppose $U_{2}$ meets $g\left(X_{1}\right)$. The first part of the proof shows that there is a component $U_{1}$ of $f_{1}^{-1}(U)$ with $g\left(U_{1}\right)=U_{2}$; and so $x_{2} \in g\left(X_{1}\right)$ and $g\left(X_{1}\right)$ is closed.

This completes our proof.

If $\left(X_{1}, f_{1}\right)$ and $\left(X_{2}, f_{2}\right)$ are two members of $C\left(X_{0}\right)$ we shall say that $\left(X_{1}, f_{1}\right) \geqq\left(X_{2}, f_{2}\right)$ if $f_{1}$ can be lifted with respect to $f_{2}$ and the lifting $\bar{f}_{1}$ is a covering map. The relation $\geqq$ is not transitive in general, as an example in $\$ 3$ shows, but it is if $X_{0}$ is uniformly covered. This follows from Lemma 2.1.

It is possible that the set $C\left(X_{0}\right)$ has a largest element relative to the relation $\geqq$. That is, there exists $(X, f)$ in $C\left(X_{0}\right)$ such that $(X, f)$ $\geqq\left(X_{1}, f_{1}\right)$ for every element $\left(X_{1}, f_{1}\right)$ of $C\left(X_{0}\right)$. We call $(X, f)$ a universal covering space of $X_{0}$.

The following theorem is in close analogy to [2, Theorem 5.1]. However, while a simply connected covering space is universal, the converse is an open question.

THEOREM 2.2. $X_{0}$ has a universal covering space if and only if $X_{0}$ is a uniformly covered space. Moreover, if $X_{0}$ is a uniformly covered 
space, and if $x_{0}$ is a point of $X_{0}$ then there exists a universal covering space $(X, f)$ and a point $x \in X$ with the following property: if $\left(X_{1}, f_{1}\right)$ is any covering space of $X_{0}$ with $x_{1}$ any point of $f_{1}^{-1}\left(x_{0}\right)$, then there exists a lifting $\bar{f}$ of $f$ with respect to $f_{2}$ such that $\bar{f}(x)=x_{1}$.

Proof. We show first that if $X_{0}$ has a universal covering space $(X, f)$ and $U$ is a neighborhood of $X_{0}$ evenly covered by $f$, then $U$ is evenly covered by $f_{1}$ where $\left(X_{1}, f_{1}\right)$ is any member of $C\left(X_{0}\right)$. Since $(X, f)$ is universal, there exists a lifting $\bar{f}$ of $f$ with respect to $f_{1}, f_{1} \circ \bar{f}$ $=f$, and $\bar{f}$ is a covering map. If $U_{\alpha}$ is an even portion of $f^{-1}(U)$ then $\bar{f}\left(U_{\alpha}\right)$ is open in $X_{1}$ because $\bar{f}$ is an open map. Also, $f_{1}^{-1}(U)=\bigcup \bar{f}\left(U_{\alpha}\right)$ where $U_{\alpha}$ ranges over all even portions of $f^{-1}(U)$, and $f_{1} \mid \bar{f}\left(U_{\alpha}\right)$ is a homeomorphism. We must show that the sets of the form $\bar{f}\left(U_{\alpha}\right)$, where $U_{\alpha}$ is an even portion of $f^{-1}(U)$, are either disjoint or coincide. Suppose that for $y_{\alpha} \in U_{\alpha}$ and $y_{\beta} \in U_{\beta}, \bar{f}\left(y_{\alpha}\right)=\bar{f}\left(y_{\beta}\right)$. Consider the homeomorphisms $f_{\alpha}=f\left|U_{\alpha}, f_{\beta}=f\right| U_{\beta}$, and the mappings $\bar{f}_{\alpha}=\bar{f} \mid U_{\alpha}$ and $\bar{f}_{\beta}=\bar{f} \mid U_{\beta}$. Both $\bar{f}_{\alpha}$ and $\bar{f}_{\beta} \circ f_{\bar{\beta}}{ }^{-1} \circ f_{\alpha}$ agree on $y_{\alpha}$ and $f_{1} \circ \bar{f}_{\alpha}=f_{1} \circ \bar{f}_{\beta} \circ f_{\bar{\beta}}{ }^{-1}$ $\circ f_{\alpha}$. By the Cancellation Lemma, $\bar{f}_{\alpha}=\bar{f}_{\beta} \circ f_{\beta}^{-1} \circ f_{\alpha}$, hence $\bar{f}\left(U_{\alpha}\right)$ $=\bar{f}\left(U_{\beta}\right)$. This completes the "only if" part of the proof.

The "if" part of the proof will follow from the stronger statement beginning with "moreover." This, however, is proved by the construction of Chevalley [1, p. 55] with one minor variation. Where he refines the topology of the constructed space until it is locally connected, we only refine it enough to make the connected disjoint even portions open. In all other respects the proof remains the same. This construction really rests on uniform even covering.

Corollary 2.3. A universal covering space is unique up to equivalence.

Proof. Select a point $x_{0}$ of $X_{0}$ and let $(X, f)$ be the universal covering space of Theorem 2.2, with $x$ in $X$ such that $f(x)=x_{0}$. Let $(Y, g)$ be another universal covering space of $X_{0}$ and $\bar{g}$ is the lifting of $g$ with respect to $f, f \circ g=g$. Select a point $y$ in $\bar{g}^{-1}(x)$. By Theorem 2.2 there is a lifting $\bar{f}$ of $f$ with respect to $g$, such that $\bar{f}(x)=y$. The identity $i$ on the connected space $Y$ and $\bar{f} \circ \bar{g}$ agree on $y$ and $g \circ i=g \circ \bar{f} \circ \bar{g}$. By the Cancellation Lemma, $\bar{f} \circ \bar{g}=i$, and $(X, f)$ and $(Y, g)$ are equivalent.

We now consider a case when the universal covering space is simply connected.

TheOREM 2.4. If $X_{0}$ is a uniformly covered space and $(X, f)$ is its universal covering space, then $X$ is simply connected if and only if for every covering space $\left(X_{1}, f_{1}\right)$ of $X,\left(X_{1}, f \circ f_{1}\right)$ is a covering space of $X_{0}$. 
Proof. In the case that $X$ is simply connected, if $\left(X_{1}, f_{1}\right)$ is a covering space of $X$ then $f_{1}$ is a homeomorphism: therefore $\left(X_{1}, f \circ f_{1}\right)$ is a covering space of $X_{0}$.

Conversely, assume that $\left(X_{1}, f_{1}\right)$ is a covering space of $X$ and that $f \circ f_{1}$ covers $X_{0}$. Choose a point $x_{0}$ in $X_{0}$ and let $x$ be the point of $X$ satisfying the "moreover" part of Theorem 2.2. Choose $x_{1}$ in $f_{1}^{-1}(x)$. By Theorem 2.2 there exists a lifting $\bar{f}$ of $f$ with respect to $f \circ f_{1}$ such that $f(x)=x_{1}$. By the Cancellation Lemma, $f_{1} \circ \bar{f}=$ identity on $X$ and $f_{1}$ is a homeomorphism. Hence, $X$ is simply connected.

Although " $\geqq$ " is not necessarily transitive if $X$ is not uniformly covered, $C(X)$ is directed relative to " $\geqq$ ". That is, given two covering spaces $(Z, h)$ and $(Y, g)$ of $X_{0}$ there is a covering space $(W, k)$ such that $(W, k) \geqq(Z, h)$ and $(W, k) \geqq(Y, g)$. It is not hard to see that $W$ can be taken to be a component of $W^{\prime}$ where $W^{\prime}$ is the subset of $Y \times Z$ consisting of the points $(y, z)$ such that $g(y)=h(z)$.

This is the construction which Novosad [3] needed to show that his set of $g$-covering spaces is directed. His proof of directedness is misleading in that he shows that every two elements have a (trivial) lower bound while an upper bound is what is needed.

3. Examples. In this section we consider some examples and miscellaneous results. We first deal with simply ordered sets. Let $X_{0}$ be a simply ordered set topologized with the order topology and connected in that topology. Note that $X_{0}$ is also locally connected so that we may use Chevalley's definition of covering space here. Say $X_{0}$ is closed if it has a maximum and a minimum. If $X_{0}$ is closed and connected then any covering by open intervals contains a finite chain of overlapping intervals joining the maximum and the minimum; hence $X_{0}$ is compact.

TheOREM 3.1. A simply ordered set connected in the order topology is simply connected.

Proof. Let $X_{0}$ be such a set. First assume that $X_{0}$ is closed, hence compact. Let $(X, f)$ be a covering space of $X_{0}$. There is a finite collection of evenly covered open intervals $I_{j}=\left(a_{j}, b_{j}\right), j=1, \cdots, n$, such that $X_{0}=\bigcup_{1}^{n} I_{j}$. We may assume that $a_{j}<b_{j-1}<a_{j+1}$. By [1, Lemma 1, p. 57] $X_{0}$ is simply connected.

Now consider the general case and let $(X, f)$ be as above. Let $x_{0}$ be a fixed point of $X_{0}$ and $x$ any other point. There is a closed, hence simply connected, interval containing $x_{0}$ and $x$ in its interior. This interval is then evenly covered by $f$, and contains an open interval $I_{x}$ also evenly covered by $f$ containing $x$ and $x_{0}$. Choose $p_{0} \in f^{-1}\left(x_{0}\right)$ 
and let $K_{x}$ be the even portion of $f^{-1}\left(I_{x}\right)$ containing $p_{0} . K=\bigcup_{x \in X_{0}} K_{x}$ is open and it is sufficient to show that $f \mid K$ is one to one. Suppose that $p_{1} \in K_{x_{1}}, p_{2} \in K_{x_{2}}$ and $f\left(p_{1}\right)=f\left(p_{2}\right)$. Since $I_{x_{1}} \cap I_{x_{2}}$ is not empty, $I=I_{x_{1}} \cup I_{x_{2}}$ is evenly covered. The even portion of $f^{-1}(I)$ containing $p_{0}$ contains both $p_{1}$ and $p_{2}$; hence $p_{1}=p_{2}$.

Theorem 3.1 implies that the real line and intervals in it are simply connected, a matter discussed by Chevalley $[1$, p. 56]. However, his argument is based on the assumption that covering spaces of the additive group of real numbers are actually covering groups. This assumption is never proved.

Theorem 3.1 also provides a source of examples of simply connected spaces which are not arcwise connected. For instance, consider a connected simply ordered set with arbitrarily small neighborhoods that are of a power higher than that of the continuum.

The following is an example of a space with no universal covering space (hence no simply connected covering space either). Let $X_{0}$ in the plane consist of a large circle $C_{0}$ with center $c_{0}$ together with smaller circles $C_{i}, i=1,2, \cdots$, outside of $C_{0}$, tangent to $C_{0}$, but not tangent to each other. Let the centers $c_{i}$ of the $C_{i}$ approach a limit point $p$ on $C_{0}$. The space $X_{0}$ is connected, and locally connected. For each $i$ there is a subspace $X_{i}$ of the Riemann surface $R_{i}$ of $\log \left(z-c_{i}\right)$ such that $\left(X_{i}, f_{i}\right)$ is a covering space of $X_{0}$ where $f_{i}$ is the restriction to $X_{i}$ of the projection from $R_{i}$ to the plane. One sees easily that no neighborhood of $p$ can be evenly covered by all the covering spaces of $X_{0}$. By Theorem 2.2, $X_{0}$ fails to have a universal covering space.

This same space also provides an example of the composition of two covering maps failing to be a covering map. There is a covering space $(Y, g)$ of $X_{0}$ where $Y$ is a subspace of the Riemann surface of $\log \left(z-c_{0}\right)$. Actually $Y$ is still a plane curve, a line with blocks of circles $C_{i j}$ all tangent to it, where $g^{-1}\left(C_{i}\right)=\bigcup_{j=-\infty}^{\infty} C_{i j}$. Roughly speaking, one obtains $Y$ by "unwinding" the circle $C_{0}$ in $X_{0} . Y$ can be arranged in the plane so that the centers of the circles $C_{k k}$ are at the points $2 \pi k$, for $k=1,2, \cdots$, on the real axis and the line, which is $C_{0}$ unwound, is below the real axis. Then there is a covering space $(W, h)$ of $Y$ in the Riemann surface of $\log \operatorname{Sin} z$, where $h$ is the restriction of the projection. The composition $h \circ g$ cannot evenly cover any neighborhood of $p$ in $X_{0}$.

Note. It has come to our attention that Theorem 2.2 and its corollary appeared in Banaschewski: Zur Existenz Von universellen Uberlagerungen, Math. Nachr. vol. 15 (1956) pp. 175-180, for connected locally connected space. While we simply apply a modification of Chevalley's method, Banaschewski's methods are entirely different. 


\section{BIBLIOGRAPHY}

1. Claude Chevalley, Theory of Lie groups, Princeton University Press, 1946.

2. C. N. Lee, Covering spaces and simple connectedness, Duke Math. J. vol. 24 (1957) pp. 547-554.

3. R. S. Novosad, Simply connected spaces, Trans. Amer. Math. Soc. vol. 79 (1955) pp. 216-228.

University OF Minnesota AND

UNIVERSITY OF WASHINGTON

\section{ONE DIMENSIONAL TOPOLOGICAL LATTICES}

LEE W. ANDERSON ${ }^{1}$

1. By a topological lattice we mean a Hausdorff space $L$ and a pair of continuous functions $\bigvee: L \times L \rightarrow L$ and $\wedge: L \times L \rightarrow L$ which satisfy the usual conditions stipulated for a lattice in Birkhoff [2, p. 18]. The purpose of this paper is to prove the

Main Theorem. A locally compact, connected, one dimensional topological lattice is a chain.

The lattice theoretic terminology used in this paper is consistent with Birkhoff [2]. The topological terms can be found in [5] or [8] with the following exceptions. If $X$ and $Y$ are sets, $X \backslash Y$ denotes the relative compliment of $Y$ with respect to $X$. If $A$ is a subset of a topological space then $A^{*}, A^{0}$ and $F(A)=A^{*} \backslash A^{0}$ denote the topological closure, interior and boundary of $A$. The symbol $\varnothing$ denotes the empty set.

We will agree that in usage of words common to topology and lattice theory, the topological meaning will take precedence. Thus to say that a subset $A$ of a topological lattice is closed means $A=A^{*}$ and not $A \wedge A \subset A$ or $A \vee A \subset A$.

If $L$ is a lattice and $A$ is a subset of $L$ we let

$$
C(A)=(A \wedge L) \cap(A \vee L) \text {. }
$$

If $A=C(A)$ we say that $A$ is a convex subset of $L$. It is clear that the set $A$ is convex if, and only if, $x \vee(y \wedge L) \subset A$ whenever $x$ and $y$ are elements of $A$ with $x \leqq y$. A topological lattice is locally convex if, and only if, whenever $x$ is an element of an open set $U$ there is an open convex set $V$ with $x \in V \subset U$.

Received by the editors October 1, 1955 and, in revised form, April 21, 1958.

1 This work was supported in part by a National Science Foundation Grant. 